\title{
Exploring on nursing educational innovation with best practice guidelines to cultivate evidence-informed practice
}

\author{
Junqiang Zhao ${ }^{1,2}$, Jinfang Wang ${ }^{1,2}$, Xinjuan Liu ${ }^{1,2}$, Liu Han ${ }^{1,2}$, Yuting Jiang ${ }^{1,2}$, Shangqian Gao ${ }^{1,2}$, Yufang Hao ${ }^{* 1,2}$ \\ ${ }^{1}$ School of Nursing, Beijing University of Chinese Medicine, Beijing, China \\ ${ }^{2}$ Beijing University of Chinese Medicine Best Practice Spotlight Organization(BPSO), Beijing, China
}

Received: June 19, 2016

DOI: $10.5430 /$ jnep.v7n1p118
Accepted: August 30, $2016 \quad$ Online Published: September 6, 2016

URL: http://dx.doi.org/10.5430/jnep.v7n1p118

\begin{abstract}
The Registered Nurses Association of Ontario (RNAO) is the professional voice of registered nurses, nurse practitioners and nursing students in the province of Ontario, Canada. RNAO's mandate is to influence and promote public health policy and clinical excellence. RNAO's best practice guideline (BPG) program established in 1999, focuses on BPG development, implementation and evaluation and has impacted clinical and academic excellence globally. The Best Practice Spotlight Organization (BPSO $®$ ) Designation, is RNAO's unique organizational knowledge exchange strategy that supports health care and academic organizations to implement and integrate multiple best practice guidelines into daily practice and education. Beijing University of Chinese Medicine (BUCM) has recently become the first academic BPSO in China to enable successful implementation of BPGs which must include not only clinical nurses but also nursing educators and students. While relatively new in implementing BPGs into the nursing curricula, BUCM School of Nursing has developed key strategies to support this work. This paper will discuss the nursing educational innovation of integrating BPGs into the curricula to cultivate evidence-based practice (EBP) at the BUCM School of Nursing from the perspectives of undergraduates, graduates and nursing educators.
\end{abstract}

Key Words: Nursing education, Best Practice Guidelines, Education innovation, Evidence-based practice

\section{INTRODUCTION}

\subsection{Importance of integration EBP into nursing educa- tion}

Integration of evidence into practice is an essential outcome for baccalaureate nursing students in this era of health care reform. ${ }^{[1]}$ In 2011, both the American Association of Colleges of Nursing (AACN) and the Institute of Medicine (IOM) emphasized that EBP is not only an important competency of baccalaureate nursing students but also should be cultivated before graduation. ${ }^{[2]}$ And the Institute of Medicine's
Health Professions Educational Summit has named EBP as one of the five core competencies for health care education. ${ }^{[3]}$ Ciliska (2005 P349) also once argued that "in another generation, all nurses graduating will be well equipped to be good consumers of research and to bring evidence to bear on decisions with practice situations". ${ }^{[4]}$ It's obvious that EBP is highly valued in recent years.

The teaching of EBP has also become increasingly popular in both undergraduate and postgraduate medical education programs worldwide. ${ }^{[5]}$ Evidence-based medicine is now a com-

\footnotetext{
*Correspondence: Yufang Hao; Email: haoyufang0903@ sina.com; Address: School of Nursing, Beijing University of Chinese Medicine, No 6. Wang Jing Zhong Huan South Road Chaoyang District, Beijing 100102, China.
} 
ponent of the foundation years training program in the UK, ${ }^{[6]}$ the focus of graduate assessment in the United States ${ }^{[7]}$ and a requirement of practicing physicians in Canada. ${ }^{[8]}$ It's self-evident that integrating EBP into medical education has become an irreversible trend.

\subsection{Value of evidence-based clinical practice guidelines in nursing education}

With strict and systematic development processes, evidencebased clinical practice guidelines (CPGs) can improve clinical nursing quality, standardize clinical nursing practice, reduce patients' nursing costs and improve clinical nurses' professional knowledge. In nursing education, such evidencebased guidelines can also be of great value for students as well as nursing educators in the following four aspects. Firstly, they enable faculty and students to renew their understanding of knowledge. Just as Dr. Sydney Burwell, Dean of Harvard Medical School, once said, half of what you are taught as medical students will in 10 years have been shown to be wrong. Guidelines are the syntheses of best available evidence, which in the case of well-developed guidelines, will be updated and renewed every 3 to 5 years as more current evidence emerges. This creates the clear understanding that knowledge is not fixed but changeable. Secondly, guidelines help cultivate critical thinking ability. With an appreciation for the finite nature of knowledge, we are all reminded to adopt a cautious attitude towards what we learned in classes or books, remembering most knowledge can quickly become irrelevant in the face of more current information. This approach to learning requires us to actively and skillfully conceptualize, apply, analyze, synthesize and evaluate information so as to foster critical thinking ability. Using this approach, it is easy to see that knowledge, skills and techniques based on well-developed best practice guidelines (BPGs) provide the most up-to-date content to support nursing education and student learning. Thirdly, recognizing that context is very critical regardless of country or jurisdiction, rigorously developed guidelines based on best evidence are relevant globally, and thus are able to support evidence-based transformation in nursing and health care internationally. Fourthly, evidence-based CPGs are a promising means to bridge the gaps between research and EBP application in practice. Students often report feeling unprepared for EBP and inadequately supported to use evidence in clinical practice. CPGs provide recommendations based on credibly appraised summaries of best evidence to assist with decisions about healthcare. Deliberate use of CPGs in curricula allows students to see how EBP learned in the classroom is merged into practice and contributes to their readiness for practice. So integration evidence-based CPGs into nursing curricula can help connect class with practice and prepare

Published by Sciedu Press nursing students to make sound clinical judgments for safe and effective practice.

\subsection{Status quo of evidence-based CPGs utilization in nursing education in China}

Successful implementation of clinical guidelines must include not only clinical nurses, but also nursing educators and students. If nursing students are to adopt clinical guidelines as a normal part of practice, the guidelines must be integrated into theory classes and reinforced during clinical experiences. ${ }^{[9]}$ Right now in China, with the high emphasize on EBP, more and more nursing colleges start evidence-based nursing courses for undergraduates and graduates. While in these courses only some basic knowledge on CPGs is described. As to the localization, utilization and sustainable use of specific guidelines, little is seen, let alone the evaluation of guideline utilization.

\subsection{Significance of localizing evidence-based CPGs into China nursing education}

There are three internationally cooperative evidence-based nursing centers in China, namely Fudan University School of Nursing, Peking University School of nursing and BUCM School of Nursing. The three sites are building their capacity in implementation science and BPG development with the assistance of world prestigious evidence-based nursing centers, such as Jonna Briggs Institute (JBI), Australia, and Registered Nurses' Association of Ontario (RNAO), Canada. Needless to say, localizing and integrating foreign rigorously developed guidelines into Chinese nursing curricula and practice settings will save time compared with developing guidelines on our own, and meanwhile boost the development of evidence-based nursing in China. That's an area needing great attention in our country. What's more important is that patients can benefit from the implementation of such guidelines based on the best evidence through receiving the best nursing care. In addition, use of evidence-based practice often results in reduction of medical, nursing and human resources costs, providing further benefit to organizations and the system.

\subsection{BPGs}

RNAO is the professional association representing registered nurses, nurse practitioners and nursing students in Ontario. It launched the Nursing Best Practice Guidelines Program in November 1999 with funding from the Government of Ontario. RNAO follows a rigorous, scientific approach to guideline development based on a systematic review, use of an expert panel, grading of the evidence, involvement of external stakeholder reviewers, and a 5-year review and revision process. ${ }^{[10]}$ Until now, RNAO has published more than 
50 BPGs and made them available at no cost online on its website (http://rnao.ca/). These guidelines has proved to be very effective, impacting both patients' and health care providers' outcomes in various settings. ${ }^{[2,11]}$

In addition, organizations are encouraged to develop an EBP culture through a process of becoming a Best Practice Spotlight Organization (BPSO). To date, there are 94 BPSOs and more than 450 BPSO sites in the world. And in 2015 and its annual general meeting, RNAO officially announced BUCM as the first BPSO center in China.

\section{NURSING EDUCATIONAL INNOVATION WITH BPGS}

\subsection{Overall project}

Localizing and integrating foreign guidelines into Chinese nursing curricula and practice settings is the goal of BUCM and its related BPSO activities. This process has been undertaken by the faculty and involves a number of steps outlined here.

\subsubsection{Guidelines translation}

BPGs translation and cultural adaption are key steps in the educational innovation of adopting BPGs into the curricula. This step requires careful planning and partnership with RNAO in order to ensure accurate translation of the selected BPGs. The specific guidelines, selected carefully by the faculty of BUCM School of Nursing in accordance with the current curricula settings, includes: Assessment and Management of Foot Ulcers for People with Diabetes, Facilitating Client Centred Learning, Toolkit: Implementation of Best Practice Guidelines, Educator's Resource: Integration of Best Practice Guidelines,Promoting Safety: Alternative Approaches to the Use of Restraints. The whole translation work is done by the faculty and graduates in BUCM School of Nursing. From the translation process, they have an overall and in-depth understanding of the standardized evidence-based guideline development procedures and the methodology beneath it. The process also includes a review by RNAO to be sure that there is no misinterpretation in the translation, ensuring a correct product is produced in Chinese. The translated BPGs, with full acknowledgement of the work of BUCM, will be hosted on the RNAO website to be freely available for use by other academic and clinical institutions in China.

\subsubsection{Web-based platform}

A web-based platform is a facilitator for communication and sharing about BPGs and their use and impact. Right now in China, WeChat is one of the most prevalent social software among citizens. We have applied a WeChat group with membership from different backgrounds like clinical nurses, nursing managers, nursing educators, nursing students, evidence-based medicine experts, etc. to disseminate the knowledge about BPGs, EBP and specific knowledge on evidence-based methodology. Until now the number of the members has reached more than 200. Here we have been uploading relevant knowledge periodically, and provide opportunities for those BPG champions and other users engage in free talk and debate about evidence-based practices, implementation science and the impact of BPG use. In addition, we are preparing for the application of a WeChat public account (where everyone using WeChat can freely follow this account, see and share the knowledge in it) to make evidencebased knowledge and the work we have done more accessible for anyone interested. At BUCM, both the WeChat public account and WeChat group are under responsibility of graduate students.

\subsubsection{Set up indicators to evaluate BPG implementation in academia}

The integration of BPGs into nursing curricula can lead to improved EBP and enhanced client and health system outcomes. While the fact is that BPGs are not always used in academic settings, and even less often evaluated for the impact on student competencies and subsequent health outcomes. There is a need to identify quality performance indicators useful to monitor the uptake and impact of EBP, and more specifically BPG integration in nursing education settings.

(1) Structure indicators Structure refers to the attributes of the setting in which education is delivered and is inclusive of the resources (facilitates, equipment, placement opportunities, etc.), attributes of the educators (knowledge, skills, values) and program structures. Based on the literature review and specific condition of BUCM School of Nursing, we identified the following structure indicators temporarily. 1) The accessibility of resources, like evidence-based material, databases, internet and teaching platforms. EBP stresses the acquirement of best evidence. With the help of these resources, we can then make evidences available. We have made questionnaires and started focused group interview to understand the status quo of students' utilization of these resources and are embedding the knowledge on the usage of specific databases and professional websites into the specific theoretical and clinical courses. 2) The financial and policy support from government and university. Leaders' attention paid to EBP can promote its successful implementation. In our year-end teaching report each year, we are required to summarize the funding we've got in EBP projects and the supportive policy and resources provided. 3) Human resources. The successful implementation of BPGs in academia requires a stable and active team. We started the team capacity building since the foundation of our evidence-based nursing center 
several years ago. By attending training programs and continuing education, now we have established a solid team with dean, senior professors specialized in clinical research, junior teachers with $\mathrm{PhD}$ degree on evidence-based medicine, graduates, undergraduates and clinical staff nurses. 4) Other structure indicators like educators' attitudes towards BPG implementation in academia, whether they are engaged, supportive, willing and enthusiastic; educators' knowledge and skills, whether they have a good understanding of EBP and are capable to explain.

(2) Process indicators Process refers to how teachinglearning transpires and the pedagogical approaches. The process indicators include three aspects. 1) The course design. The collaboration among faculty to design courses and discussion on the strategies on embedding BPGs into curricula; adding new objectives relating to EBP implementation to course syllabi. 2) Various forms of pedagogical practice. Different instructional designs like peer teaching, face-to-face seminars, and journal clubs, etc. and teaching techniques like problem-based or case-based learning approaches are resorted to to make the class more active. Besides, sharing the evidence-based content with student in the theoretical courses (for instance the history of EBP, evidence-based principles and elements, EBP models, steps of EBP, levels of Evidence, critical appraisal, clinical guidelines, etc.). 3) Students' formative evaluation. Focus group interviews are recommended to obtain students' attitudes towards BPGs. ${ }^{[9]}$ Tests or exams, evidence-base related assignments and projects (like analyze abstracts, critical appraisals) are also the ways.

(3) Outcome indicators Outcomes refer to the end points of the educational innovation including: learners, educators and whole organizations outcomes. 1) For learners utilizing different approaches (the quantitative approach, for instance, the Evidence-based Practice Questionnaire, EBPQ, Developing Evidence-based Practice Questionnaire, DEBPQ, Chinese Critical Thinking Disposition Inventory, CTDICV. Those are all mature questionnaires widely used with high reliability and validity. And qualitative approach such as interviews, observations) to understand students' reaction or attitudes towards EBP implementation, whether they will apply evidence-based clinical guidelines into their work and research, whether they become more confident in care practice; the knowledge and skills on EBP, whether they have a good mastery of EBP steps, whether they are able to find evidence, apply evidence; their practice change after the educational innovations, have they ever used evidence-based guidelines in client care or teaching process, when they encounter professional questions, what kind of resources they often turn to for help. 2) For faculty, it's a routine for School of Nurs-

Published by Sciedu Press ing to hold semester-end meeting and hand in semester-end BPSO-related report to BPSO headquarter. This meeting is regarded as an exchange program for all faculty to summarize the gain and loss, success and failure in this semester's teaching work. And the report covered by the chiefs of each departments is also an approach to evaluate the work they have done. 3) For the whole college, we will assess the whole educational achievements like the funding we got, projects applied, papers published, professional textbooks edited, the collaboration and long-term partnership with clinic, etc.

\subsection{Undergraduates}

\subsubsection{Curricula designs}

Different courses lay emphasis on the utilization of different guidelines. We plan to integrate the BPGs as follows: Facilitating Client Centred Learning and the Educator's Resource: Integration of Best Practice Guidelines into the "Introduction of Nursing" course; Assessment and Management of Foot Ulcers for People with Diabetes into the "Internal Medication Nursing" course; Promoting Safety: Alternative Approaches to the Use of Restraints into the "Basic Nursing" course; and Toolkit: Implementation of Best Practice into the "Nursing Research" course, etc. As to the strategies to embed these BPGs into the specific pedagogical practice, we resort to various forms of activities to make the class more vibrant, for instance, peer teaching, seminars, roleplay problem-based learning, etc. Besides, share the specific recommendations, assessment tools, and professional association websites in those BPGs with students in the teaching PowerPoint. Demonstrate the specific approaches on where and how to find high quality evidence (eg., papers in different databases, guidelines online). Discuss the differences between recommendations and the corresponding content in textbook and explain the reasons beneath it so as to convey the idea that knowledge is not fixed but changeable. In clinical classes, we also require students to preview the guideline materials, ask questions relating to the recommendations, and complete leave assignments to critically analyze the applicability of selected BPGs in the long-term facilities. ${ }^{[12]}$ Students are also expected to integrate them into their clinical work.

One side benefit to having our students work directly with the English language BPGs is that given the high quality of professional nursing language, their use helps our students better understand the scientific language of nursing and health care as it is expressed in English. We have recommended use of the BPGs in English classes where our students are encouraged to read, translate, and understand guidelines. 


\subsubsection{Summer vacation class and nursing research club}

At the beginning of the summer vacation, BUCM School of Nursing provides lectures on EBP for undergraduates routinely since 2014. Professional evidence-based nursing experts are invited to give lectures on nursing research. Students have great passion on those courses and each year more than 70 students participate in it. We have also set up a nursing research club where we the BPG champions (mainly undergraduates in school of nursing) can get together. We have planned series of activities for champions, like inviting teachers and graduates to give lectures about EBP and BPGs; tackling undergraduates' nursing research issues; offering suggestions and instructions on manuscript preparation and publication. We hope to build an academic nursing atmosphere and evidence-based culture and facilitate the research ability for undergraduates through the summer vacation class and research club.

\subsection{Graduates}

\subsubsection{Curricula designs}

It is imperative for all master's prepared nurses to become competent, active consumers of the "best available evidence" that informs clinical decision-making. ${ }^{[13]}$ So along with evidence-based nursing theoretical course, we leave 4 class hours to lecture on the RNAO "implementation toolkit", knowledge-to-action model and BPG implementation science (these knowledge are all extracted from Toolkit: Implementation of Best Practice Guideline, an evidence-based practice guideline developed by RNAO), mainly including, how to adapt foreign high-quality guidelines into local context, identify facilitators and barriers on guideline implementation, select and tailor implementation interventions, monitor and evaluate guideline use and sustain the achievements, etc. And we think it would be helpful to provide specific comparisons between potentially confusing concepts, such as practice standards and clinical guidelines in this class, how each are developed and used to advance safe health care and clinical practice. ${ }^{[9]}$ Besides, teachers are required to embed the evidence-based practice project conducted by our college into the theoretical course, share with students the challenges and achievements they encountered in the process and the tips while undertaking an evidence-based nursing practice project.

\subsubsection{In charge of the WeChat public account and WeChat group}

Graduate students are the leading group that uploads BPGrelated knowledge into the WeChat public account and WeChat group and manage its operation. They provide topics and materials to undergraduate users and assist in identifying evidence-based solutions to the clinical issues undergradu- ates encounter. Prior to uploading, graduates should have a good mastery of the knowledge they provide. It is an effective approach to enhance graduates own understanding of BPGs , meanwhile boost the self-learning and team discussion among champions following the WeChat public account and WeChat group.

\subsubsection{Assisting undergraduates research work}

Graduate students also provide support to the nursing research club. In the process of assisting undergraduates with their research work, graduate students can improve and strengthen their own knowledge on nursing research.

\subsubsection{Transfer BPGs into health education resources}

We are also working on the goal of supporting an evidencebased culture in hospitals and communities through providing and supporting evidence-based health education for clients. As part of this goal we are working to develop health education resources in plain language that follow the recommendations in the Client-Centered Learning BPG. This will enable patients and community citizens to more easily learn and master information about their specific health concerns on health promotion and disease prevention.

\subsection{Nursing educators}

\subsubsection{Set up the project foundation in nursing college}

The BUCM School of Nursing has established a foundation specially for evidence-based nursing research. The purpose of the foundation is to support and encourage nursing faculty and students in our academic setting to study more about EBP and BPGs and do more research in this field. We will provide opportunities for faculty to attend evidence-based nursing training programs, offer publishing and expert consultation fee. The foundation mainly cover the following research fields, like systematic review, evidence integration, guideline implementation and so on.

\subsubsection{Series of teachers' evidence-based nursing salon}

For faculty we have launched a series of teacher's evidencebased practice salons. The salon is an EBP reference group and in the salon teachers share experiences on writing evidence-based papers, methods on integrating evidence and implementing guidelines, and also share strategies on embedding guidelines into their courses and lectures. We encourage clinical faculty to participate in the salon as it is critical to enhance opportunities for interaction and reinforce the importance of integrating the evidence and classroom learning into practice.

\section{DiscuSSION AND CONCLUSION}

This work embarked upon by BUCM is totally unprecedented for BUCM and indeed in academia in China, as we take 
a leading role in integrating BPGs into nursing education. BPGs implementation in School of Nursing has, to some extent, changed nursing educators' teaching pattern from teacher-centered to students-centered and changed students' practice pattern from textbook-based to evidence-based and facilitate the critical thinking abilities of nursing students. We will continue refinement and improvement of our curricula and the nursing science courses to fully incorporate EBP concepts throughout. Predictably the process will be fraught with twists and turns, however, it is imperative that we continue to keep our eye on the overall outcomes of this work which are: to impact student learning, faculty development, clinical nursing practice, ultimately better health of the

\section{REFERENCES}

[1] Bloom KC, Olinzock BJ, Radjenovic D, et al. Leveling EBP content for undergraduate nursing students. J Prof Nurs. 2013; 29(4): 217 224. PMid: 23910923 http://dx.doi.org/10.1016/j.profn urs. 2012.05.015

[2] Davies B, Edwards N, Ploeg J, et al. Insights about the process and impact of implementing nursing guidelines on delivery of care in hospitals and community settings. BMC Health Serv Res. 2008; 8: 29. PMid: 18241349 http://dx.doi.org/10.1186/1472-696 3-8-29

[3] Institute of Medicine Committee on the Health Professions Education, S. 2003 In Greiner, A. C. \& Knebel, E. (Eds.) Health Professions Education: A Bridge to Quality. Washington (DC), National Academies Press (US) Copyright 2003 by the National Academy of Sciences. All rights reserved.

[4] Ciliska D. Educating for evidence-based practice. J Prof Nurs. 2005; 21(6): 345-350. PMid: 16311229 http://dx.doi.org/10.1016 /j.profnurs.2005.10.008

[5] Crilly M, Glasziou P, Heneghan C, et al. Does the current version of 'Tomorrow's Doctors' adequately support the role of evidence-based medicine in the undergraduate curriculum? Med Teach. 2009; 31(10): 938-944. PMid: 19877868 http://dx.doi.org/10.3109/01421 590903199650

[6] Foundation Programme Committee of the Academy of Medical Royal Colleges. Curriculum for the foundation years in postgraduate education and training. UK: Departments of Health. 2007. population in China. We look forward to a bright future for nursing education and for nursing and health care in China.

\section{ACKNOWLEDGements}

The authors wish to thank Dr. Irmajean Banjok, Tasha Penney from Registered Nurses' Association of Ontario, Ontario Canada and Dr. JoAnne MacDonald, Dr. Patti Hansen Ketchum, Dr. Joanne Whitty Rogers from St. Francis Xavier University, Nova Scotia Canada who facilitated and supported the completion of the paper, especially in the part of indicators construction.

\section{CONFlicts OF InTEREST Disclosure}

The authors declare that there is no conflict of interest.
[7] Association of American Medical Colleges (AAMC). Medical school program evaluation survey: Graduation questionnaire 2008. Washington, DC: AAMC. 2008.

[8] Frank JR. The CanMEDS 2005 physician competency framework: Better standards. Better physicians. Better care. Canada: The Royal College of Physicians and Surgeons of Canada. 2005. Available from: http://www.ub.edu/medicina_unitateducaciomedi ca/documentos/CanMeds.pdf

[9] Higuchi KA, Cragg CE, Diem E, et al. Integrating clinical guidelines into nursing education. Int J Nurs Educ Scholarsh. 2006; 3: 12. PMid: 16646939 http://dx.doi.org/10.2202/1548-923X.1223

[10] Grinspun D. Cómo transformar la enfermería a través del conocimiento basado en la evidencia. Enfermería Familiary Comunitaria. 2013; 1: 104-105 [in Spanish].

[11] Higuchi KS, Davies BL, Edwards N, et al. Implementation of clinical guidelines for adults with asthma and diabetes: a three-year follow-up evaluation of nursing care. J Clin Nurs. 2011; 20(9-10): 1329-1338. PMid: 21492279 http://dx.doi.org/10.1111/j.1365-2702 . 2010.03590.x

[12] Cable-Williams B, Rush J, Mowry A, et al. An educational innovation to foster evidence-informed practice. J Nurs Educ. 2014; 53(3): S55-S58. PMid: 24530015 http://dx.doi.org/10.3928/014 84834-20140217-06

[13] Sackett DL, Strauss SE, Richardson WS, et al. Evidence-based medicine: How to practice and teach EBM (2nd ed.). London, UK: Churchill-Livingstone. 2000. Available from: http://www.clinch em.org/content/47/9/1747.full 